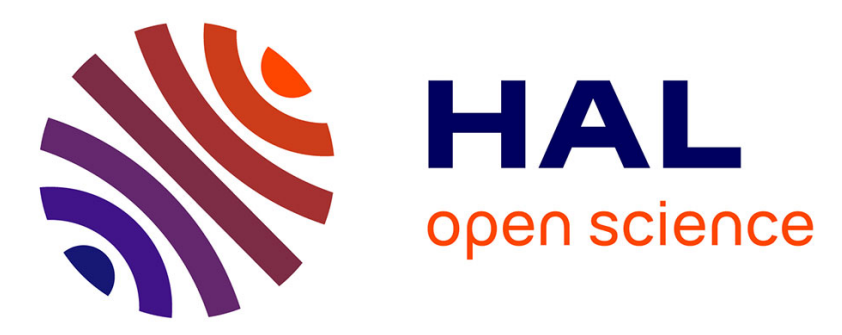

\title{
Pairing-up viologen cations and dications: a microscopic investigation of van der Waals interactions
}

Christophe Gourlaouen, Sergi Vela, Sylvie Choua, Mathilde Berville, Jennifer Wytko, Jean Weiss, Vincent Robert

\section{- To cite this version:}

Christophe Gourlaouen, Sergi Vela, Sylvie Choua, Mathilde Berville, Jennifer Wytko, et al.. Pairingup viologen cations and dications: a microscopic investigation of van der Waals interactions. Physical Chemistry Chemical Physics, 2018, 20 (44), pp.27878-27884. 10.1039/C8CP04543G . hal-01993519

\author{
HAL Id: hal-01993519 \\ https://hal.science/hal-01993519
}

Submitted on 24 Jan 2019

HAL is a multi-disciplinary open access archive for the deposit and dissemination of scientific research documents, whether they are published or not. The documents may come from teaching and research institutions in France or abroad, or from public or private research centers.
L'archive ouverte pluridisciplinaire HAL, est destinée au dépôt et à la diffusion de documents scientifiques de niveau recherche, publiés ou non, émanant des établissements d'enseignement et de recherche français ou étrangers, des laboratoires publics ou privés. 


\section{Physical Chemistry Chemical Physics}

We have presented the Graphical Abstract text and image for your article below. This brief summary of your work will appear in the contents pages of the issue in which your article appears.

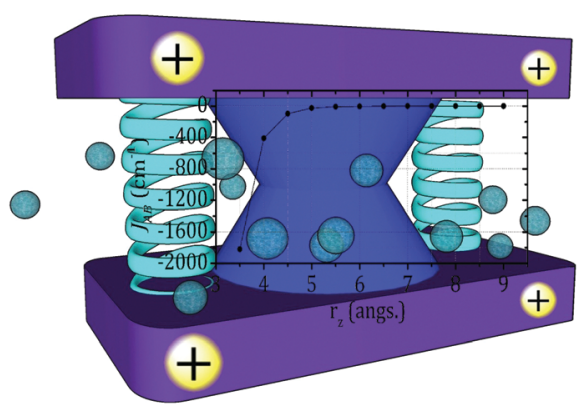

Pairing-up viologen cations and dications: a microscopic investigation of van der Waals interactions

Christophe Gourlaouen,* Sergi Vela, Sylvie Choua, Mathilde Berville, Jennifer A. Wytko, Jean Weiss and Vincent Robert*

Polarizability and simultaneous environment effects overcome Coulomb repulsions.

Please check this proof carefully. Our staff will not read it in detail after you have returned it.

Please send your corrections either as a copy of the proof PDF with electronic notes attached or as a list of corrections. Do not edit the text within the PDF or send a revised manuscript as we will not be able to apply your corrections. Corrections at this stage should be minor and not involve extensive changes.

Proof corrections must be returned as a single set of corrections, approved by all co-authors. No further corrections can be made after you have submitted your proof corrections as we will publish your article online as soon as possible after they are received.

Please ensure that:

- The spelling and format of all author names and affiliations are checked carefully. You can check how we have identified the authors' first and last names in the researcher information table on the next page. Names will be indexed and cited as shown on the proof, so these must be correct.

- Any funding bodies have been acknowledged appropriately and included both in the paper and in the funder information table on the next page.

- All of the editor's queries are answered.

- Any necessary attachments, such as updated images or ESI files, are provided.

Translation errors can occur during conversion to typesetting systems so you need to read the whole proof. In particular please check tables, equations, numerical data, figures and graphics, and references carefully.

Please return your final corrections, where possible within $\mathbf{4 8}$ hours of receipt, by e-mail to: pccp@rsc.org. If you require more time, please notify us by email. 


\section{Funding information}

Providing accurate funding information will enable us to help you comply with your funders' reporting mandates. Clear acknowledgement of funder support is an important consideration in funding evaluation and can increase your chances of securing funding in the future.

We work closely with Crossref to make your research discoverable through the Funding Data search tool (http://search.crossref.org/funding). Funding Data provides a reliable way to track the impact of the work that funders support. Accurate funder information will also help us (i) identify articles that are mandated to be deposited in PubMed Central (PMC) and deposit these on your behalf, and (ii) identify articles funded as part of the CHORUS initiative and display the Accepted Manuscript on our web site after an embargo period of 12 months.

Further information can be found on our webpage (http://rsc.li/funding-info).

\section{What we do with funding information}

We have combined the information you gave us on submission with the information in your acknowledgements. This will help ensure the funding information is as complete as possible and matches funders listed in the Crossref Funder Registry.

If a funding organisation you included in your acknowledgements or on submission of your article is not currently listed in the registry it will not appear in the table on this page. We can only deposit data if funders are already listed in the Crossref Funder Registry, but we will pass all funding information on to Crossref so that additional funders can be included in future.

\section{Please check your funding information}

The table below contains the information we will share with Crossref so that your article can be found via the Funding Data search tool. Please check that the funder names and grant numbers in the table are correct and indicate if any changes are necessary to the Acknowledgements text.

\begin{tabular}{|l|l|l|l|}
\hline Funder name & $\begin{array}{l}\text { Funder's main } \\
\text { country of origin }\end{array}$ & $\begin{array}{l}\text { Funder ID } \\
\text { (for RSC use only) }\end{array}$ & Award/grant number \\
\hline & & & \\
\hline
\end{tabular}

\section{Researcher information}

Please check that the researcher information in the table below is correct, including the spelling and formatting of all author names, and that the authors' first, middle and last names have been correctly identified. Names will be indexed and cited as shown on the proof, so these must be correct.

If any authors have ORCID or ResearcherID details that are not listed below, please provide these with your proof corrections. Please ensure that the ORCID and ResearcherID details listed below have been assigned to the correct author. Authors should have their own unique ORCID iD and should not use another researcher's, as errors will delay publication.

Please also update your account on our online manuscript submission system to add your ORCID details, which will then be automatically included in all future submissions. See here for step-by-step instructions and more information on author identifiers.

\begin{tabular}{|l|l|l|l|}
\hline First (given) and middle name(s) & Last (family) name(s) & ResearcherID & ORCID iD \\
\hline Christophe & Gourlaouen & & 0000-0002-2409-2849 \\
\hline Sergi & Vela & & \\
\hline Sylvie & Choua & & \\
\hline Mathilde & Berville & & \\
\hline Jennifer A. & Wytko & & $0000-0001-5370-4253$ \\
\hline Jean & Weiss & & $0000-0001-7753-8958$ \\
\hline Vincent & Robert & & $0000-0003-3021-204 X$ \\
\hline
\end{tabular}




\section{Queries for the attention of the authors}

Journal: PCCP

Paper: $\mathbf{c 8 c p 0 4 5 4 3 g}$

Title: Pairing-up viologen cations and dications: a microscopic investigation of van der Waals interactions

For your information: You can cite this article before you receive notification of the page numbers by using the following format: (authors), Phys. Chem. Chem. Phys., (year), DOI: 10.1039/c8cp04543g.

Editor's queries are marked on your proof like this Q1, Q2, etc. and for your convenience line numbers are indicated like this $5,10,15, \ldots$

Please ensure that all queries are answered when returning your proof corrections so that publication of your article is not delayed.

\begin{tabular}{|l|l|l|}
\hline $\begin{array}{c}\text { Query } \\
\text { reference }\end{array}$ & \multicolumn{1}{|c|}{ Query } & Remarks \\
\hline Q1 & $\begin{array}{l}\text { Please confirm that the spelling and format of all author names } \\
\text { is correct. Names will be indexed and cited as shown on the } \\
\text { proof, so these must be correct. No late corrections can be } \\
\text { made. }\end{array}$ & \\
\hline
\end{tabular}


Cite this: DOI: $10.1039 / c 8 c p 04543 g$

\title{
Pairing-up viologen cations and dications: a microscopic investigation of van der Waals interactions
}

\author{
Christophe Gourlaouen, (D) *a Sergi Vela, ${ }^{a}$ Sylvie Choua, ${ }^{b}$ Mathilde Berville, ${ }^{c}$ \\ Jennifer A. Wytko, (D) ${ }^{c}$ Jean Weiss (D) ${ }^{c}$ and Vincent Robert (D) *a
}

\begin{abstract}
The microscopic origin of van der Waals- and magnetic-interactions in 4,4' methyl viologen cationbased units $\left(\mathrm{MV}^{+} \bullet\right.$ and $\mathrm{MV}^{2+}$ ) was inspected using wave function (variational DDCl and perturbative MP2, CASPT2) and density functional theory (DFT) calculations. The analysis deepens the comprehension of the magnetic behavior of experimental bis-viologen cyclophanes $\left([C Y C]^{2(\bullet \bullet}\right)$, in which the $\mathrm{MV}^{+\bullet}$ units are connected through alkyl linkers of different lengths. The formation of the so-called long-multicenter bonds in such radical dimers, responsible for the quenching of the magnetic response, was analyzed in $\left[\mathrm{MV}_{2}\right]^{2(+\bullet)}$. Dynamical correlation effects, accessible from second-order perturbation corrections, were decisive in observing a bonding regime characterized by an equilibrium distance of $3.3 \AA$ and a $45 \mathrm{~kJ}$ $\mathrm{mol}^{-1}$ dissociation energy. At larger intermolecular distances, our calculations on $\left[\mathrm{MV}_{2}\right]^{2(+\bullet)}$ indicate that the singlet and triplet states are energetically competing (i.e. weak exchange interactions, $J_{A B}$ ). Despite the absence of any clear bonding regime at the MP2 level, the puzzling association of two di-cations into $\left[\mathrm{MV}_{2}\right]^{4+}$ is anticipated at $3.3 \AA$ using weakly screened point charges $(\varepsilon=1.5)$ to account for the Coulomb interactions between the solvated subunits. The main conclusion is that both dispersion interactions and environment effects are required to overcome the Coulomb repulsion associated with doubly-charged species. All these data provide some complementary insights into the nature and amplitude of interactions between cation and dication units, and their relevance in various experimental

manifestations.
\end{abstract}

Received 18th July 2018, Accepted 23rd October 2018

DOI: $10.1039 / \mathrm{c} 8 \mathrm{cp} 04543 \mathrm{~g}$

rsc.li/pccp

\section{Introduction}

Organic-based radicals have attracted much attention due to their ubiquitous character, being capable to act as spin 40 holders, ${ }^{1}$ switchable materials ${ }^{2,3}$ and binding ligands, ${ }^{4}$ not to mention their ability to form three dimensional networks. ${ }^{5}$ From their flexible electronic structure, unusual properties including conductivity, magnetism, spin crossover or charge ordering have been observed and stimulated intensive work. ${ }^{6-11}$

45 Furthermore, changes in the spin states can be controlled by manipulating the unpaired electrons, taking advantage of their intrinsic degree of freedom. ${ }^{12}$

Bistability in organic radical species is largely dominated by their tendency to dimerize, quenching the desired property of

${ }^{a}$ Laboratoire de Chimie Quantique, UMR 7111, CNRS-Université de Strasbourg, 4 rue Blaise Pascal, F-67000 Strasbourg, France.E-mail: velallausi@unistra.fr

${ }^{b}$ Laboratoire de Propriétés Optiques et Magnétiques des Architectures Moléculaires, UMR 7111, CNRS-Université de Strasbourg, 4 rue Blaise Pascal, F-67000 Strasbourg, France

$55{ }^{c}$ Laboratoire de Chimie des Ligands à Architecture Contrôlée, UMR 7111, CNRSUniversité de Strasbourg, 4 rue Blaise Pascal, F-67000 Strasbourg, France interest while forming a diamagnetic ground state. ${ }^{13,14}$ In some cases, the (apparently) non-dimerized paramagnetic phase has also been found to be the result of a fast dimerization thus giving the impression that they are, indeed, not dimerized. ${ }^{15}$ Such dimerization is also active in some charged (monooxidized or reduced) species, where the repulsive Coulomb contribution is overcome by interactions that have been attributed to overlaps between the $\pi$-SOMOs (singly-occupied molecular orbitals) centred on each radical cation. ${ }^{16}$ This type of interaction presents spectroscopic similarities to covalent bonds but differs in their nature, strength, and equilibrium distance. ${ }^{17-20}$ In this context, paraquat cations (e.g. 1,1'dimethyl-4,4'-bipyridinium) within the viologen family, are particularly attractive platforms as the formation of so-called $\pi$-bonds is likely to reversibly generate dimeric units under redox stimulus. ${ }^{21}$ The bipyridinium radical-cations are held together in sandwich-like arrangements displaying much shorter distances $(c a$. $3.0 \AA$ ) than the standard $\pi-\pi$ stacking distance (3.6 ̊). More recently, synthetic strategies have been developed to vary the number of alkyl linkers between viologen units (cyclophane structures $[\mathrm{CYC}]^{2(+\bullet)}$ leading to compounds $\mathbf{1}$, 


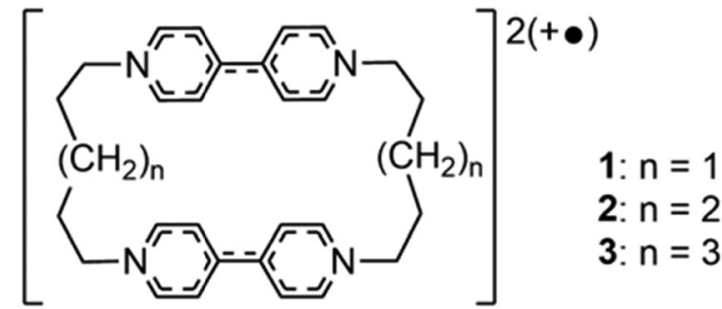

Fig. 1 Cyclophane structure $\left([\mathrm{CYC}]^{2(+\bullet)}\right)$ consisting of two viologen cation radical units linked together through alkyl chains of variable size. Compounds $\mathbf{1}, 2$ and 3 correspond to $n=1,2$ and 3, respectively.

2 and 3, see Fig. 1), and to ultimately modulate the host-guest properties. ${ }^{21}$ In the cyclophane units, the cation radicals are atoms (see Fig. 1).

The $\pi$-dimerization of these species was analyzed by means of electrochemistry, vis/NIR spectroscopy and spectroelectrochemistry. In the meantime, the electronic structures were tentatively inferred by EPR spectra. A well-resolved EPR signal could only be observed for 2 in fluid solution revealing the presence of a biradical with a strong antiferromagnetic interaction $\left(\mathrm{ca} .-734 \mathrm{~cm}^{-1}\right) .^{22}$ Unfortunately, it was not possible to measure a clear EPR signal for compounds $\mathbf{1}$ and 3. Quite interestingly, the crystal structure of 2 was obtained for the tetra-cation $[\mathrm{CYC}]^{4+}, 23$ a regime where doubly-charged organic subunits are held together. Density functional theory (DFT) calculations were then performed on isolated cyclophanes in a model of water, stressing a marked propensity towards antiferromagnetism. ${ }^{22}$ More recently it has been suggested that large dispersion forces dominate the bonding regime in viologen cation-radical $\pi$-dimer (so-called pimer). ${ }^{24}$ The analysis was carried out from DFT calculations including dispersion corrections and concluded on the competition between covalent and dispersion attractions with Coulomb repulsions.

The heart of the current investigation is whether the strong antiferromagnetism and packing are a consequence of the screened charges in $[\mathrm{CYC}]^{2(+\bullet)}$ and $[\mathrm{CYC}]^{4+}$ units or must be attributed to dispersion interactions. Therefore, we felt that a

microscopic analysis would be instructive to rationalize both the $\pi$-dimerization and the magnetic properties of this family of cations and radical cations. Starting from experimental X-ray data on $[\mathrm{CYC}]^{2(+)}$ (see Fig. 1), viologen radical-based compounds were examined using DFT and wave function calculawith the aim of understanding the origin of the interaction at van der Waals distances. Based on the structure of the wave function, the relative importance of the so-called ionic and neutral forms is discussed in comparison to the $\mathrm{C}_{2} \mathrm{H}_{6}$ molecule, bonded molecule. Then, a similar inspection was carried out on the tetracationic form $\left[\mathrm{MV}_{2}\right]^{4+}$. The puzzling binding of two doubly-charged $\mathrm{MV}^{2+}$ units, as observed in the crystal structure of 2, deserves particular attention in order to understand its nature. Finally, the magnetic properties of $\left[\mathrm{MV}_{2}\right]^{2(+\bullet)}$ were examined based on calculations of magnetic exchange couplings on the optimized dimer geometries. Due to the flexible nature of the alkyl linkers in the cyclophanes, different conformations were expected to be present in solution. These inspections allow us to understand how the relative positions of the $\mathrm{MV}^{+} \bullet$ units modulate the nature and amplitude of the magnetic interactions.

In this framework, our intention is to examine the dimerization process holding both viologen units at rather short distances in comparison with standard $\pi-\pi$ stacking distances.

\section{Technical background}

For $\left[\mathrm{MV}_{2}\right]^{2(+\bullet)}$ dication species, results were first obtained from complete active space self-consistent field (CASSCF) calculations including two electrons in two molecular orbitals (MOs), namely CAS[2,2]. All these calculations were performed using the MolCAS 7.2 package. $^{25}$ The atoms were described with extended basis sets of ANO-RCC types, [4s3p2d1f] for C and N atoms, and [2s1p] for the $\mathrm{H}$ ones. ${ }^{26}$ Based on the CAS[2,2]SCF singlet ground state wave function, complementary second-order perturbation calculations (CASPT2) were performed. Total energies were evaluated at the CAS[2,2]PT2 level with respect to $r_{z}$, the distance between two viologen units, and the structure of the wave function was analyzed. Such a framework allows one to build the wave function and to extract the information relative to the important dynamical correlation effects. The active space was extended to CAS[6,6] with no quantitative change in the calculated CASPT2 binding energies (42 $\mathrm{kJ} \mathrm{mol}^{-1}$ for $\mathrm{CAS}[6,6] \mathrm{PT} 2$ and $45 \mathrm{~kJ} \mathrm{~mol}^{-1}$ for CAS[2,2]PT2). Calculations were restricted to MP2 type (Möller-Plesset) for the closed-shell tetra-cation $\left[\mathrm{MV}_{2}\right]^{4+}$ analogues.

Second, the nature of the radical interaction was examined through a configuration interaction (CI) restricted to single excitations (CAS[2,2]SCF $+\mathrm{S})$. These CI calculations were carried out with the CASDI code ${ }^{27}$ to retrieve the leading charge redistribution beyond the mean field CASSCF picture. For comparison with a prototype of covalent bond, we performed CAS[2,2]SCF $+\mathrm{S}$ calculations for the $\mathrm{C}_{2} \mathrm{H}_{6}$ ethane molecule at equilibrium and stretched carbon-carbon distances (1.54 and $3.0 \AA$ A ) using a [3s2p1d] and [2s1p] basis sets for carbon and hydrogen atoms, respectively. At $3.0 \AA$, the active space consists in the bonding and antibonding $\sigma$-type MOs based on the $\mathrm{CH}_{3}{ }^{\bullet}$ SOMOs. This set of MOs was then used as a starting set to converge the CAS[2,2] solution at a $1.54 \AA$ carbon-carbon distance. From the presence of a center of inversion, the active MOs are the in-phase (u) and out-of-phase (g) linear combinations of the SOMOs localized on each individual unit. It is wellknown that the CAS[2,2]SCF $+\mathrm{S}$ singlet wave function mostly reads $\Psi=\lambda|g \bar{g}|-\mu|\mathrm{u} \bar{u}|$ where $\mathrm{g}$ and $\mathrm{u}$ are the canonical bonding and antibonding MOs (see Fig. 2a and b). A unitary transformation allows one to rewrite $\Psi$ in the local atomic basis set to evaluate the ratio between the so-called ionic and neutral form as $\rho=(\lambda-\mu) /(\lambda+\mu){ }^{28}$ The diradical character is featured by a reduction of $\rho$, and can be considered as a measure of covalency to be compared with the so-called $\pi$-dimerization mechanism. 


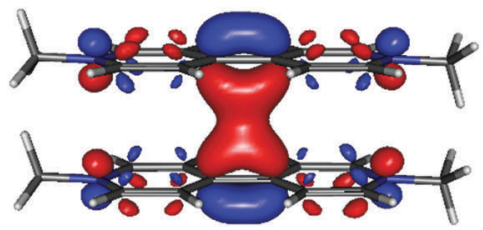

(b)

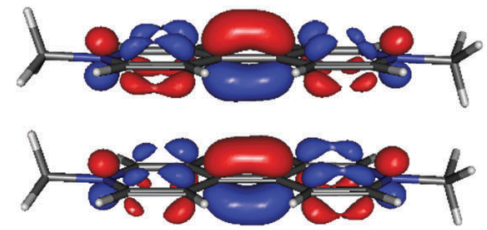

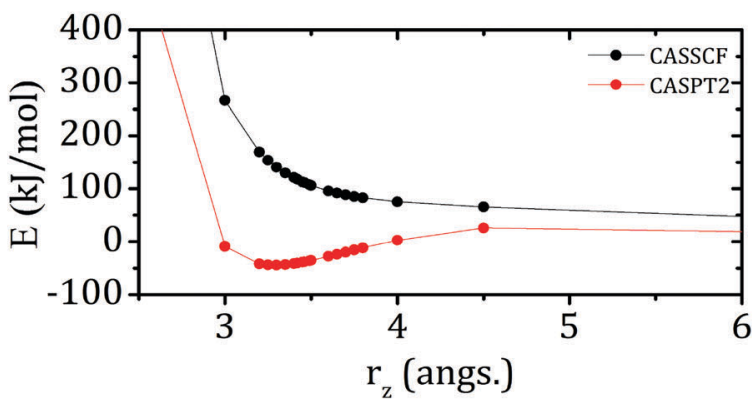

(c) CAS[2,2]SCF and CAS[2,2]PT2 Fig. 2 Triplet canonical CAS[2,2]SCF MOs (a) bonding, $g$ symmetry, and (b) antibonding, u symmetry of $\left[\mathrm{MV}_{2}\right]^{2(+\bullet)}$. (c) CAS[2,2]SC
$\left[\mathrm{MV}_{2}\right]^{2(+\bullet)}$ energies $\left(\mathrm{KJ} \mathrm{mol}^{-1}\right)$ with respect to the interplanar distance $r_{z}(\AA)$. The energy reference is taken at $r_{z}=10 \AA$, arbitrarily.

Third, in the presence of two unpaired electrons in a dimer of viologen cation radicals, low-lying singlet (total spin $S=0$ ) difference defines the so-called magnetic exchange coupling constant $2 J_{\mathrm{AB}}=E_{\mathrm{S}}-E_{\mathrm{T}}$ ruling the Heisenberg spin-Hamiltonian $H=-2 J_{\mathrm{AB}} S_{1} S_{2}$, where $S_{1}$ and $S_{2}$ are the $3 \mathrm{D}$-spin operators acting on each subunit, and are associated with spin values $\frac{1}{2}$. Two numerical approaches were used to evaluate the exchange coupling constants. Difference dedicated configuration interaction (DDCI) calculations were carried out using the CASDI code. ${ }^{27}$ Such methodology has proven to provide not only very accurate estimates of magnetic exchange coupling constants but also microscopic interpretations. ${ }^{29,30}$ The atomic basis sets were safely reduced down to [3s2p1d] for carbon and nitrogen atoms, and [2s] for hydrogen ones based on similar inspections carried out on verdazyl organic radicals. ${ }^{31}$ Throughout the DDCI calculations, the triplet CAS[2,2] MOs were used. We checked that any modification of the set of MOs leads to negligible changes in the singlet-triplet energy difference. To reduce the computational cost, the two alkyl chains of the cyclophane units were substituted by four methyl groups (yielding pairs of cation viologen units, referred to as $\left[\mathrm{MV}_{2}\right]^{2(+\bullet)}$ and $\left[\mathrm{MV}_{2}\right]^{4+}$, respectively). We confirmed that this simplification has a limited impact on the calculated energy differences. Quantitatively, the triplet-singlet energy difference at the DDCI level are 4697 and $4794 \mathrm{~cm}^{-1}$ for the full and simplified diradicals at $2.9 \AA$ distance, respectively (i.e. less than $3 \%$ with the fact that such alkyl chains are poor mediators of magnetic interaction in diradicals $[\mathrm{CYC}]^{2(\bullet \bullet)}$.

Taking advantage of the relative low-cost of the DFT calculations, different degrees of freedom (interplanar distance, slippages and rotations) were considered to derive magnetostructural analysis. The broken-symmetry (BS) approach ${ }^{32,33}$ provides the energies of a fictitious open-shell state and a high-spin state. Different strategies were proposed to evaluate the exchange-coupling constant from these values. ${ }^{34}$ The relative singlet-triplet energy differences between DDCI and DFT calculations are less than $5 \%$. All calculations were performed using the B3LYP functional (restricted or unrestricted), and the atoms were described by TZVP basis sets as implemented in Gaussian $09^{35}$ in gas phase.

\section{Results and discussion}

Wave function theory based calculations were performed (i) to analyze the bonding mechanism, (ii) to trace the stability of dication $[\mathrm{CYC}]^{2(+\bullet)}$ and tetra-cation $[\mathrm{CYC}]^{4+}$ cyclophane units, and (iii) to provide exchange coupling constant values in the diradical $[\mathrm{CYC}]^{2(+\bullet)}$.

\section{Nature of the interaction between two $\mathrm{MV}^{+\bullet}$ units}

Despite the well-accepted terminology "pimer", the interaction has been viewed as resulting, at least in part, from $\sigma$-overlap between $\pi$ systems. ${ }^{16}$ To clarify the nature of the interaction between the viologen cation radicals $\mathrm{MV}^{+}$, the variations of CAS[2,2]SCF and CAS[2,2]PT2 total energies were followed with respect to the intra-planar distance $\left(r_{z}\right)$, maintaining a perfect stacking between the viologen units. As expected, the active MOs are the in-phase $(\mathrm{u})$ and out-of-phase $(\mathrm{g})$ linear combinations of the SOMOs localized on each individual unit (see Fig. 2a and b). Fig. 2c displays the calculated energy of the ground state singlet performed at various levels (CAS[2,2]SCF and CAS[2,2]PT2). To compare the energy variations, the $r_{z}=10$ $\AA$ values was set to zero as a common reference. Despite the presence of two unpaired electrons, the interaction cannot be understood from a "two electrons in two MOs" picture, that might be qualitatively acceptable for neutral radicals. ${ }^{36}$ The absence of any binding regime at the CASSCF level suggests that the interaction does not fall in the traditional covalent bond scenario. The one-electron picture constructed on the SOMOs overlap is not appropriate to qualitatively account for the weak bond formation. As soon as second-order perturbation corrections are included, the interactions between units considerably change. A marked minimum is observed for $r_{z}=$ $3.3 \AA$ (see Fig. 2), associated with a binding interaction of $45 \mathrm{~kJ}$ $\mathrm{mol}^{-1}$. In the present case, the bonding character is reached 
1 with dynamical correlation effects, similarly to what is wellknown between closed-shell systems such as benzene rings (i.e. dispersion-like interactions). At this stage, one should mention that shorter equilibrium distances ( $c a .3 .0 \AA$ ) have been reported experimentally. ${ }^{16}$ Solvent molecules in solution as well as counter-anions in the solid state are likely to screen the Coulomb repulsions, allowing closer inter-molecular contacts.

To gain further insight into the nature of this interaction, the ratio between the ionic and neutral amplitudes (see com-

10 putational details) was calculated from the CAS[2,2]SCF $+\mathrm{S}$ wave function at the equilibrium distance $r_{z}=3.3 \AA$. The $\rho$ value was then compared to the values calculated for the reference $\mathrm{C}_{2} \mathrm{H}_{6}$ molecule at equilibrium $(1.54 \AA)$ and stretched $(3.0 \AA)$ carbon-carbon distances. The wave function provides the $\sigma / \sigma^{*}$ 15 electronic distribution resulting from the $\mathrm{CH}_{3}{ }^{\bullet}$ SOMOs overlap. From the wave function analysis, this quantity amounts to 0.27 in $\left[\mathrm{MV}_{2}\right]^{2(+\bullet)}$. This is significantly smaller than the values of 0.84 and 0.32 found in $\mathrm{C}_{2} \mathrm{H}_{6}$ at equilibrium and stretched carboncarbon distances, respectively. From this inspection, the con$\left[\mathrm{MV}_{2}\right]^{2(+\bullet)}$ cation as compared to the value in $\mathrm{C}_{2} \mathrm{H}_{6}$ at equilibrium distance, and still smaller than the one in the dissociated regime. This observation is consistent with the prime role played by the electronic correlation effects. This highlights the radical character with vanishingly-small electron delocalization in the interaction between the two $\mathrm{MV}^{+}$units.

\section{Binding of two $\mathrm{MV}^{2+}$ units?}

We then moved to the tetracationic derivatives $\left[\mathrm{MV}_{2}\right]^{4+}$ which 30 was reported in the crystal structure of $2 .{ }^{21}$ Multiply-charged species are not so scarce in solid state chemistry. In such species, the chemical bonding in an ionic solid is dominated by electrostatic interactions. Even at the electrostatic level, the Coulomb interactions between units are screened thanks to the environment. ${ }^{37,38}$ In addition, the intrinsic polarizabilities of the building blocks are likely to give rise to stabilizing interactions. From previous investigations, and in the light of our current results on the $\left[\mathrm{MV}_{2}\right]^{2(\bullet)}$ cation, the dispersion forces significantly overcome the Coulomb repulsion since a $45 \mathrm{~kJ}$ $0 \mathrm{~mol}^{-1}$ binding interaction is calculated in vacuum (see previous section). How strong are dispersion interactions in this class of compounds, where highly charged species have been observed? It was rather tempting to assess the ability of such contributions to generate a tetra-cation from two closed-shell $45 \mathrm{MV}^{2+}$ units in the absence of any counter ion. Thus, secondorder Möller-Plesset calculations (MP2) were carried out by varying the interplanar distance $r_{z}$. In sharp contrast with the parent $\left[\mathrm{MV}_{2}\right]^{2(+\bullet)}$, no binding regime is observed for $\left[\mathrm{MV}_{2}\right]^{4+}$ : the Coulomb repulsion exceeds the dispersion interactions in the matter the phase (condensed: liquid or solid), solvent molecules as well as counter ions act as mediators of the $\pi$ dimerization. We can evaluate their participation through the introduction of a dielectric constant $\varepsilon$. The electrostatic interactions are effectively reduced as a consequence of the charge screening. Therefore, the Coulomb repulsion was first

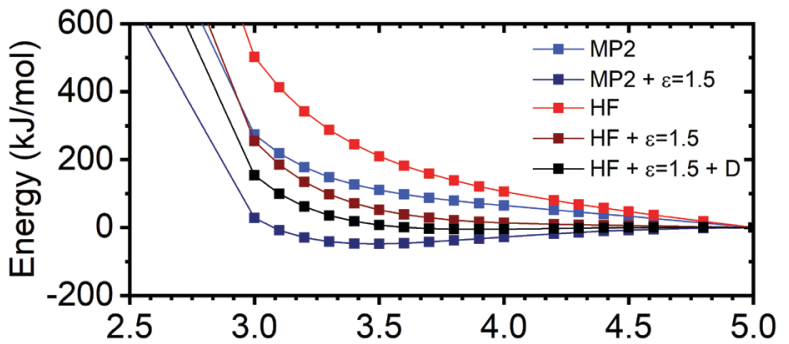

Fig. 3 Dissociation curves of $\left[\mathrm{MV}_{2}\right]^{4+}$ at $\mathrm{HF}$ and MP2 level of theory and influence of a dielectric constant $(\varepsilon)$ on the electrostatic interactions considering two $2+$ point charges and on the correlation contributions (D). For each curve, the reference energy is set to zero for $r_{z}=5 \AA$.

corrected to account for these effects through the following term: $\left(-4 / r_{z}\right)(1-1 / \varepsilon)$, and $\varepsilon$ was varied starting from an $\varepsilon=1$ value (i.e., vacuum). Formally, this quantity corresponds to the change in the electrostatic repulsion between two $2+$ charges upon going from vacuum to a dielectric medium characterized by $\varepsilon$. As soon as a rather weak $\varepsilon=1.5$ value is reached, the MP2 potential energy curve suddenly exhibits a clear minimum for $r_{z}$ $=3.5 \AA$ (Fig. 3, curve MP2 $+\varepsilon$ ). The resulting binding energy is of the order of $40 \mathrm{~kJ} \mathrm{~mol}^{-1}$, which is a rather large value considering the nature of the system. At this stage, one may first question the amplitude of the binding energy compared to the value found for the dication $\left[\mathrm{MV}_{2}\right]^{2(+\bullet)}$. Evidently, the interplanar distance being very similar, the screened Coulomb correction is four times larger than it is in the dication. Thus, one may then argue that this correction may mask the contributions of the dispersion-like interactions. However, we checked that the potential energy curve remains dissociative at the Hartree-Fock level even if these contributions are included (Fig. $3, \mathrm{HF}+\varepsilon$ curve). Despite the questionable validity of such a description at rather short distances, the impact of the electrostatic continuum was also estimated by screening the correlation contributions, a second order effect. The energy differences between the MP2 and HF values were renormalized by $1 / \varepsilon^{2}$ (see Fig. $3, \mathrm{HF}+\varepsilon+D$ curve). Such procedure underestimates the correlation effects since both inter- and intrasubunit contributions are screened. Still, a bonded geometry is observed for $r_{z}=3.9 \AA$ (and a non-negligible binding energy of ca. $6 \mathrm{~kJ} \mathrm{~mol}^{-1}$ ). Finally, our views were complemented using HF calculations in the presence of a PCM, followed by a perturbative treatment. The PCM polarizes the MOs and account for the MOs distortion induced by the solvent. Such procedure allows one to grasp part of the dispersion-induced charge rearrangements, the importance of which has already been stressed. ${ }^{39}$ In water, a binding regime is observed, which confirms that both screening and dispersion are required to hold the $2+$ units together. In other words, the viologen polarization remains decisive to establish a bonding regime, which cannot be reached using a mean-field description.

\section{Magneto-structural analysis}

Starting from the DFT optimized geometry of $\left[\mathrm{MV}_{2}\right]^{2(+\bullet)}$, characterized by an inter-molecular distance $r_{z}$ of $3.6 \AA$ and a co- 
1 facial arrangement $\left(r_{x}=0\right)$, the $J_{\mathrm{AB}}$ values were calculated for two translations, the interplanar distance $\left(r_{z}\right)$ and the longitudinal slippage $\left(r_{x}\right)$, and two rotations, $\alpha$ around the stacking ( $z$ axis) and $\beta$ around the $x$ axis (see Fig. 4). Other variables were found to be less relevant. We did not intend to provide a full potential energy surface of the low-lying singlet and triplet states, but rather to evaluate the relative changes in the exchange-coupling constant in the vicinity of the equilibrium geometry. As expected, the $J_{\mathrm{AB}}$ intensity increases exponentially from $\mathrm{ca}$. -90 to $0 \mathrm{~cm}^{-1}$ a). Such behaviour was analysed in similar compounds and can be attributed to the exponential variation of the SOMOs overlap and hopping integrals. ${ }^{31,40}$ At this stage, let us mention that the available crystal structures for compounds $\mathbf{1}$ and $\mathbf{3}$ exhibit interplanar distances which are larger than $4.8 \AA$. Thus, one would expect quasi-degenerate singlet and triplet, as reflected by a negligibly small $J_{\mathrm{AB}}$ value. The non-linear response of $J_{\mathrm{AB}}$ with respect to $r_{z}$ indicates that thermal oscillations would result in a significantly different mean value for $J_{\mathrm{AB}}$ due to dynamic effects, making it more antiferromagnetic than the value obtained using a static picture. $^{41}$ In compound 2 , such phenomena would support the EPR measurements of a $J_{\mathrm{AB}}$ value of $-734 \mathrm{~cm}^{-1} .^{22}$ In contrast, the $J_{\mathrm{AB}}$ variations along the longitudinal slippage exhibit a double minimum curve (see Fig. $4 \mathrm{~b}$ ). The first one is observed for $r_{x}=0 \AA$, with a $J_{\mathrm{AB}}$ value reaching $c a .-700 \mathrm{~cm}^{-1}$.
For this "on-top" geometry, the SOMOs overlap is optimal through facing pairs of pyridinium rings. Since the superexchange mechanism grows with the square of the overlap the resulting AFM contribution is then maximized. When $r_{x}$ deviates from zero, the super-exchange mechanism is disrupted and $J_{\mathrm{AB}}$ increases until $r_{x}$ reaches $4 \AA$. For this value, the overlap between units is restricted to a single pair of pyridinium rings (see Fig. $4 \mathrm{~b}$, right inset) and, thus, the resulting $J_{\mathrm{AB}}$ value should be roughly a quarter of the initial one. In this case, it is $-250 \mathrm{~cm}^{-1}$.

Let us now examine the effects of rotations on the exchange coupling constant. Along the $z$ axis ( $\alpha$ parameter in Fig. 4e), the curve exhibits two wells for $\alpha=0^{\circ}$ and $\alpha=90^{\circ}$ associated with $J_{\mathrm{AB}}$ values of -1400 to $-800 \mathrm{~cm}^{-1}$, respectively (see Fig. 4c), and a maximum for $\alpha=40^{\circ}$. As previously mentioned, these variations reflect the modulation of the overlap between the SOMOs. Due to the intrinsically delocalized nature of the latter (see Fig. 2a), $\alpha=90^{\circ}$ corresponds to a local minimum for the exchange coupling constant. Upon variation of $\beta$ (see Fig. $4 \mathrm{~d}$ ), $J_{\mathrm{AB}}$ follows an exponential law, being directly controlled by the superexchange decay along this distortion. ${ }^{42,43}$ To conclude, the nature of the coupling between $\mathrm{MV}^{+} \bullet$ units mostly falls in the antiferromagnetic regime, with rather large amplitude variations over the explored coordinates. It is only for $r_{x}=2 \AA$ and $r_{x}$ = $6 \AA$ A slippage values that the singlet and triplet states become quasi-degenerate. (c)

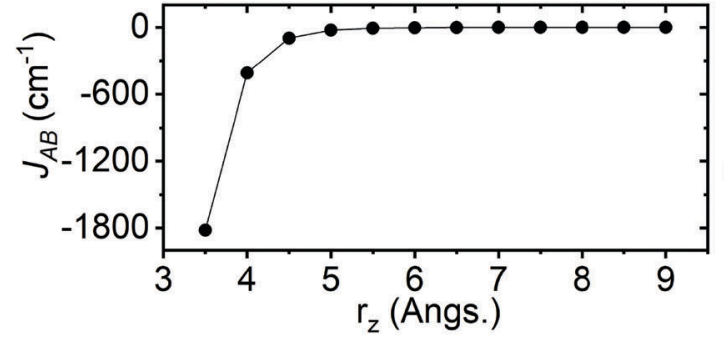

(b)

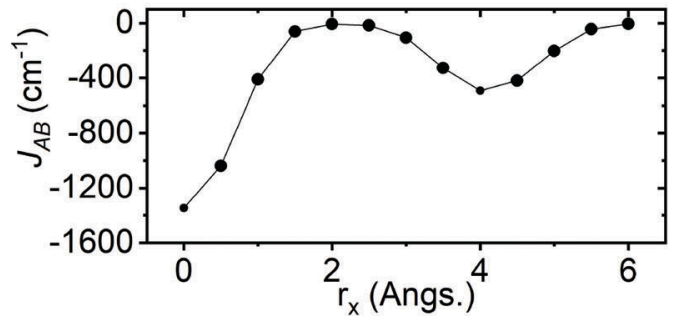

(d)
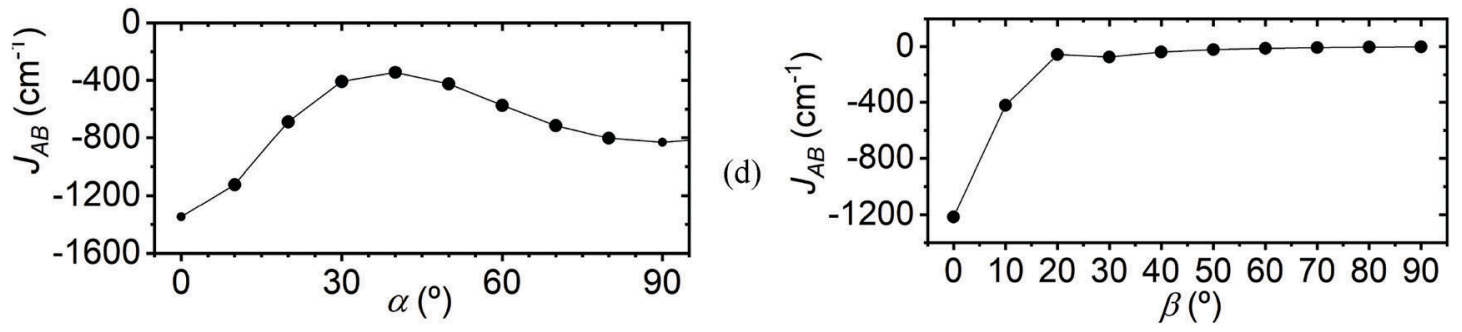

(e)

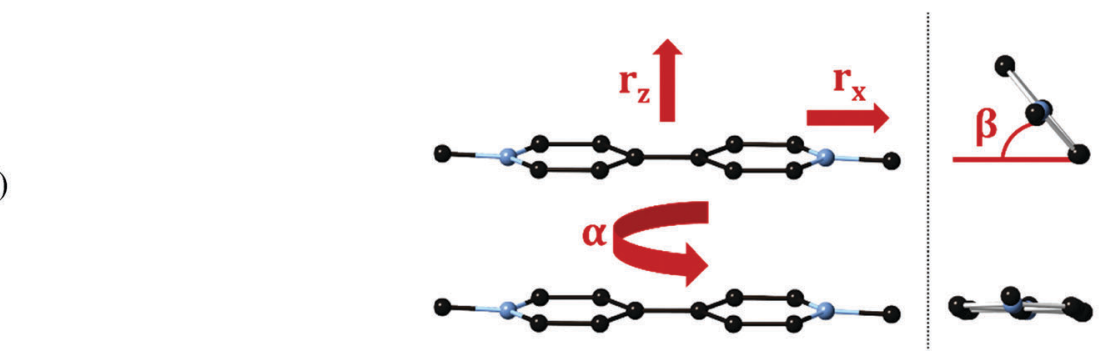

Fig. 4 Evolution of $J_{A B}$ values upon a change in the interplanar distance $\left(r_{z}, a\right)$, the longitudinal slippage $\left(r_{x}, b\right)$, and a rotation along the stacking $(\alpha, c)$ and longitudinal $(\beta, d)$ axes, defined in (e) computed in DFT level of theory. The interplanar distance is set at $3.6 \AA$ for $b, c$ and d, which is the equilibrium distance in these conditions. 


\section{Conclusions}

In the present work, we shed some light on the electronic structure of $[\mathrm{CYC}]^{2(\bullet)}$ architectures using quantum chemistry calculations. Leaving out the alkyl linkers, we first showed the persistence of the radical nature of the $\mathrm{MV}^{+} \cdot$ units even when van der Waals interactions occur. This was done by computing and analyzing the CASSCF and CASPT2 dissociation curves. In particular, we stressed the negligible weight of the charge transfer electronic configurations (i.e. ionic forms) even at short distances, suggesting that the radical character of each $\left[\mathrm{MV}_{2}\right]^{2(\bullet \bullet)}$ unit was preserved. Thus, the interaction in $[\mathrm{CYC}]^{2(\bullet \bullet)}$ architectures cannot be restricted to a one-electron picture of overlapping SOMOs. In solvent conditions where additional screening effects should be included, aggregation is observed at shorter distances as reflected by strong antiferromagnetic interactions. This led us to examine the tetracationic derivative $\left[\mathrm{MV}_{2}\right]^{4+}$. The appearance of a binding regime results from the competition between dispersion interactions and moderately screened Coulomb repulsions. The introduction of an $\varepsilon=1.5$ constant value to account for the presence of solvent molecules and/or anions significantly stabilizes a pair of $\mathrm{MV}^{2+}$ cations. This observation supports that the combination of dispersion interactions and of screening are mandatory to allow the binding between the doubly-charged species.

From a magnetic point of view, the alkyl linkers are not relevant when computing the $J_{\mathrm{AB}}$ values (DDCI calculations), justifying the transferability of the results obtained for $\left[\mathrm{MV}_{2}\right]^{2(+\bullet)}$ to the target $[\mathrm{CYC}]^{2(+\bullet)}$ architectures. Other deformations from the perfect stacking reduce the strength of $J_{\mathrm{AB}}$, with a dominating antiferromagnetic behavior. From available X-ray data, one can conclude that isolated dimers cannot create strong magnetic interactions. Even if the larger alkyl linkers provide the cyclophane more flexibility, the geometries that can be potentially adopted by these compounds are associated with weak magnetic interactions. Therefore, the only consistent explanation for the presence of strong antiferromagnetic couplings is that distances smaller than the calculated equilibrium distance of $3.3 \AA$ can be reached between two different $[\mathrm{CYC}]^{2(\bullet)}$ units. Despite the positive charges in both units, solvent molecules can be mediators of the $\pi$-dimerization, as extensively reported experimentally ${ }^{13,14}$ and rationalized computationally. ${ }^{44,45}$ In this class of compounds, screening and dispersion effects act hand-in-hand to generate unusual regimes.

\section{Conflicts of interest}

There are no conflicts to declare.

\section{Acknowledgements}

We thank the HPC centre of Strasbourg for computing time. We are grateful to Dr Manuel Ruiz-Lopez for fruitful discussions.

\section{References}

1 I. Ratena and J. Veciana, Chem. Soc. Rev., 2012, 41, 303.

2 R. G. Hicks, Nat. Chem., 2011, 3, 189.

3 W. Fujita and K. Awaga, Science, 1999, 286, 261.

4 W. Fujita and K. Awaga, J. Am. Chem. Soc., 2001, 123, 3601.

5 I. Alcon, D. Reta, I. d. P. R. Moreira and S. T. Bromley, Chem. Sci., 2017, 8, 1027.

6 P. Kaszyński, C. P. Constantinides and V. G. Young, Angew. Chem., Int. Ed., 2016, 55, 11149.

7 C. P. Constantinides, A. A. Berezin, G. A. Zissimou, M. Manoli, G. M. Leitus, M. Bendikov, M. R. Probert, J. M. Rawson and P. A. Koutenis, J. Am. Chem. Soc., 2014, 136, 11906.

8 C. M. Robertson, A. A. Leitch, K. Cvrkalj, R. W. Reed, D. J. T. Myles, P. A. Dube and R. T. Oakley, J. Am. Chem. Soc., 2008, 130, 8414.

9 C. M. Robertson, A. A. Leitch, K. Cvrkalj, D. J. T. Myles, R. W. Reed, P. A. Dube and R. T. Oakley, J. Am. Chem. Soc., 2008, 130, 14791.

10 L. Norel, J.-B. Rota, L.-M. Chamoreau, G. Pilet, V. Robert and C. Train, Angew. Chem., Int. Ed., 2011, 50, 7128.

11 J. M. Rawson, A. Alberola and A. Whalley, J. Mater. Chem., 2006, 16, 2560.

12 C. Herrmann, G. C. Solomon and M. A. Ratner, J. Am. Chem. Soc., 2010, 132, 3682.

13 S. V. Rosokha and J. K. Kochi, J. Am. Chem. Soc., 2007, 129, 828.

14 J.-M. Lü, S. V. Rosokha and J. K. Kochi, J. Am. Chem. Soc., 2003, 125, 12161.

15 S. Vela, F. Mota, M. Deumal, R. Suizu, Y. Shuku, A. Mizuno, K. Awaga, M. Shiga, J. J. Novoa and J. Ribas-Arino, Nat. Commun., 2014, 5, 4411.

16 C. Kahlfuss, A. Milet, J. Wytko, J. Weiss, E. Saint-Aman and C. Bucher, Org. Lett., 2015, 17, 4058.

17 J. S. Miller and J. J. Novoa, Acc. Chem. Res., 2007, 40, 189.

18 K. E. Preuss, Polyhedron, 2014, 79, 1.

19 R. Zhao and R.-Q. Zhang, Phys. Chem. Chem. Phys., 2016, 18, 25452.

20 M. Capdevila-Cortada, J. Ribas-Arino and J. J. Novoa, J. Chem. Theory Comput., 2014, 10, 650.

21 M. Berville, L. Karmazin, J. A. Wytko and J. Weiss, Chem. Commun., 2015, 51, 15772.

22 M. Berville, S. Choua, C. Gourlaouen, C. Boudon, L. Ruhlmann, C. Bailly, S. Cobo, E. Saint-Aman, J. Wytko and J. Weiss, Chem. Phys. Chem., 2017, 18, 796.

23 CCDC 1051151.

24 M. R. Geraskina, A. S. Dutton, M. J. Juetten, S. A. Wood and A. H. Winter, Angew. Chem., Int. Ed., 2017, 56, 9435.

25 F. Aquilante, L. De Vico, N. Ferré, G. Ghigo, P.-Å. Malmqvist, P. Neogrády, T. B. Pedersen, M. Pitonak, M. Reiher, B. O. Roos, L. Serrano-Andrés, M. Urban, V. Veryazov and R. Lindh, J. Comput. Chem., 2010, 31, 224.

26 B. O. Roos, R. lindh, P.-A. Malmqvist, V. Veryazov and P.O. Widmark, J. Phys. Chem. A, 2005, 109, 6575.

27 N. Ben Amor and D. Maynau, Chem. Phys. Lett., 1998, 286, 211. 
28 J.-P. Malrieu, C. Angeli and R. Cimiraglia, J. Chem. Educ., 2008, 85, 150.

29 J.-B. Rota, C. J. Calzado, C. Train and V. Robert, J. Chem. Phys., 2010, 132, 154702.

530 O. Oms, J.-B. Rota, L. Norel, C. J. Calzado, H. Rousselière, C. Train and V. Robert, Eur. J. Inorg. Chem., 2010, 5373.

31 J.-B. Rota, B. Le Guennic and V. Robert, Inorg. Chem., 2010, 49, 1230.

32 L. Noodleman and E. R. Davidson, Chem. Phys., 1986, 109, 131.

33 L. Noodleman, J. Chem. Phys., 1981, 74, 5737.

34 F. Neese, Coord. Chem. Rev., 2009, 253, 526.

35 M. J. Frisch, G. W. Trucks, H. B. Schlegel, G. E. Scuseria, M. A. Robb, J. R. Cheeseman, G. Scalmani, V. Barone, G. A. Petersson, H. Nakatsuji, X. Li, M. Caricato, A. Marenich, J. Bloino, B. G. Janesko, R. Gomperts, B. Mennucci, H. P. Hratchian, J. V. Ortiz, A. F. Izmaylov, J. L. Sonnenberg, D. Williams-Young, F. Ding, F. Lipparini, F. Egidi, J. Goings, B. Peng, A. Petrone, T. Henderson, D. Ranasinghe, V. G. Zakrzewski, J. Gao, N. Rega, G. Zheng, W. Liang, M. Hada, M. Ehara, K. Toyota, R. Fukuda, J. Hasegawa, M. Ishida, T. Nakajima, Y. Honda, O. Kitao, H. Nakai, T. Vreven, K. Throssell, J. A. Montgomery, Jr., J. E. Peralta, F. Ogliaro, M. Bearpark, J. J. Heyd, E. Brothers, K. N. Kudin, V. N. Staroverov, T. Keith, R. Kobayashi, J. Normand,
K. Raghavachari, A. Rendell, J. C. Burant, S. S. Iyengar, J. Tomasi, M. Cossi, J. M. Millam, M. Klene, C. Adamo, R. Cammi, J. W. Ochterski, R. L. Martin, K. Morokuma, O. Farkas, J. B. Foresman and D. J. Fox, Gaussian 09, Revision D.01, Gaussian, Inc., Wallingford CT, 2009.

36 B. D. Koivisto, A. S. Ichimura, R. McDonald, M. T. Lemaire, L. K. Thompson and R. G. Hicks, J. Am. Chem. Soc., 2006, 128, 690.

37 S. Vela, A. Sopena, J. Ribas-Arino, J. J. Novoa and M. Deumal, Chem. - Eur. J., 2014, 20, 7083.

38 A. Domingo, M. Verot, F. Mota, C. de Graaf, J. J. Novoa and V. Robert, Phys. Chem. Chem. Phys., 2013, 15, 6982.

39 N. Ferri, R. A. DiStasio, A. Ambrosetti and A. Tkatchenko, Phys. Rev. Lett., 2015, 114, 176802.

40 O. Kahn and B. Briat, J. Chem. Soc., Faraday Trans. 2, 1976, 72, 268.

41 S. Vela, M. Deumal, M. Shiga, J. J. Novoa and J. Ribas-Arino, Chem. Sci., 2015, 6, 2371.

42 C. J. Calzado, J. Cabrero, J.-P. Malrieu and R. Caballol, J. Chem. Phys., 2002, 116, 3985.

43 C. J. Calzado, J. Cabrero, J.-P. Malrieu and R. Caballol, J. Chem. Phys., 2002, 116, 2728.

44 I. Garcia-Yoldi, J. S. Miller and J. J. Novoa, J. Phys. Chem. A, 2007, 111, 8020.

45 M. Capdevila-Cortada, J. Ribas-Arino, A. Chaumont, G. Wipff and J. J. Novoa, Chem. - Eur. J., 2016, 22, 17037. 